\title{
Research on Value Orientation and Consciousness Reconstruction in the Era of Intellectual Media
}

\author{
Ge Ziqi ${ }^{1}$ \\ ${ }^{1}$ School of Humanities and Law, Northeastern University, Shenyang, Liaoning, 110819 \\ 734380844@qq.com
}

\begin{abstract}
The relationship between artificial intelligence technology and media is getting closer and closer. Although the intelligent information recommendation algorithm in the intelligent media era has greatly improved the coverage and arrival rate of information dissemination, the algorithm service based on big data and artificial intelligence is expanding day by day. Algorithms are infiltrating into our daily life and social operation in a quiet and rapid way. Focusing on the value bias of the media under the background of big data's artificial intelligence, this paper discusses the transformation of artificial intelligence technology to the media information industry from a rational point of view. The technological values in the intelligent era not only influence the production mechanism and news communication order of the news media, but also have a profound impact on the media literacy of the audience. Due to the technical, unilateral and hidden characteristics of algorithm power, there are still many defects, such as the lack of comprehensive and objective cognition, inability to identify human emotional changes and needs, ethical and legal gaps and so on. It aggravates the inequality, hinders the free choice and increases the insecurity, which makes people worry about whether the power of the algorithm can be controlled or not. From the philosophical aspect of intelligent technology, values are embedded in intelligent technology in a way that how to define rationality. Only by establishing an intelligent communication algorithm platform with value rationality as the value, the media information industry can return to paying attention to the development of human itself in the development of digitalization and intelligence, and combine algorithm recommendation with manual editing. Improve the audience's media literacy and other aspects. Therefore, the study of information values in the intelligent era needs to examine intelligent technology in the context of media information dissemination, and then explore an important research point in the era of new media technology.
\end{abstract}

Keywords: ; refactoring; The value principle; Information dissemination

\section{INTRODUCTION}

When the human society faces enormous social changes brought about by the wide range of uncertainty[1], the issue of values is discussed because of the old structure and behavior. For artificial intelligence, in a narrow sense, intelligence refers to artificial intelligence. Professor NilsJ Nilsson of Stanford University believes that artificial intelligence is an activity dedicated to making machines intelligent. For the current development of artificial intelligence technology, Dr. Jerry Kaplan of Stanford University pointed out that artificial intelligence has made breakthroughs in two aspects: one is machine learning, and artificial intelligence collects data from the same field[2]. When the volume of data is large enough to find some patterns and make full use of these patterns to predict or classify the data, the final system result is unpredictable. Second, manual workers can tirelessly complete all kinds of work in a complex and changeable environment, and this kind of system lacks common sense and general intelligence[3].

According to the current references, $50 \%$ of the literature on values is concentrated in the fields of higher education, ideological and political education, pedagogy, vocational education, secondary education and so on. This is obviously different from the previous studies on values, which are mainly distributed in philosophy, ethics, economics, pedagogy, anthropology, sociology, social psychology and other fields. There are many problems in a small number of studies. For example, when some scholars studied the relationship between the changes of Chinese values and the 
media in the transitional period, they extracted some important issues that need to be clarified: first, in the field of Chinese news and communication, the concept of values has not been fully discussed, and scholars in the field of news and communication have not studied the exact meaning of values when studying the issues related to media and values. Therefore, there are problems of uncertainty, instability and disunity in the meaning of values. Second, the research is lack of empirical evidence, and it is often only a general discussion, and the very complex issues such as "the relationship between media and the change of values" are not fully demonstrated, but with a relatively simple logic[4].

Into wisdom media era, the technique of artificial intelligence algorithms and big data applications and constantly improve[5], while greatly increasing coverage and the arrival rate of information transmission. But there are still some defects in its developing process, the need for positive refactoring.

\section{THE INNOVATION EFFECT OF ARTIFICIAL INTELLIGENCE TECHNOLOGY ON NEW MEDIA PATTERN}

\subsection{A new way of writing characters}

In recent years, artificial intelligence technologies represented by deep learning, reinforcement learning and transfer learning have made continuous breakthroughs in text processing, audio processing and image processing, which have opened the prelude for machines to engage in content production.

The application of intelligent technology mainly focuses on the thinking dimension of intelligent technology, including network information retrieval and mining, image processing and video processing, natural language processing and machine translation and so on. These intelligent technologies are both irrational and rational, and their main purpose is to assist or dominate the information retrieval, cleaning, coding, translation, clustering and other links in the information production process, focusing on improving the computing power and production efficiency of information production. For example, the data news team of the Associated Press uses machine learning techniques in judging the emotions of interviewees and discovering news clues from huge amounts of data. AutomatedInsights and other institutions apply natural language technology to automatic writing of structured messages and abstract extraction of unstructured data. Future lab of
ReynoldsJournalismInstitute and others use voice processing technology in the voice interface of news consumption, automatic dictation of interview recordings, automatic capturing of video quotes and making titles; some media organizations use visual information technology for facial expression recognition, satellite high-definition image recognition, automatic tagging of videos and photos, etc. The German EarthTV satellite television company and many media companies use robots for automated writing and unconventional angle shooting of drones. Artificial intelligence technology has its unique ability when it is used in the field of news editing, which is different from manual editing.

Previously, the machines could only handle news from relatively fixed content structures such as finance, sports and earthquakes. Nowadays, machine writing has also begun to be applied in people's livelihood, science and technology and other fields. For example, xiao Nan, a robot reporter of Southern Metropolis Daily, has been writing in people's livelihood, current politics and news.

\subsection{Efficient distribution of information}

The recommendation system of a platform is usually not an overall recommendation algorithm, but a recommendation platform, which has many different algorithms to ensure that when one set of algorithms is blocked, it can be switched to other algorithms to continue to run. In the process of operation, the adjustment of any element may affect the final recommendation result of the whole system. The factors that affect and may change the recommendation results include not only feature extraction, recommendation architecture, algorithm parameters, rule strategies, but also the environment and behavior of individual users, the content circulation of the information platform and so on.

The recommendation system has a certain degree of uncertainty in the process of operation, and no one can clearly judge whether a piece of information can be sent to a user's terminal, because the algorithm system has the characteristics of dynamic and self-operation, that is to say, people's power in news distribution has been transferred to artificial intelligence. It changes from intelligent thinking mode to behavior mode, including machine deep learning, opinion mining, social network path analysis, user profile and other technologies. Baidu search and Jinri Toutiao personalized recommendation are the practical applications of artificial intelligence in the field of information 
distribution. The in-depth learning machine is based on big data automatically fetching, matching and presenting information from the database according to user retrieval keywords or personal attributes and behavior records, and then intelligently and efficiently distribute information according to its behavior path on the social network. The purpose is to ensure a high effective coincidence rate between the information and the needs of users, and to reduce the release of information that users are not interested in.

\section{DEFECTS OF INTELLIGENT INFORMATION ALGORITHM}

\subsection{Emotion recognition}

Algorithm technology shows strong data processing ability, but at the same time, it cannot recognize human subtle emotional changes, artificial intelligence algorithm technology itself is unable to express meaning independently, it has no emotion and expression ability, and it cannot identify and judge human emotional changes. In the process of the vigorous development of artificial intelligence algorithm technology, algorithm technology is also known as network neural network, which can reach audience groups and individuals at all levels, and can reach every network nerve ending. However, the problem of algorithm technology's lack of attention and response to human emotion is also highlighted. For example, at the beginning, we talked about the annoyance caused by the recommendation of algorithmic technology.

Smart technology have same thinking and behavior of the application of two dimensions, but as a result of the interactive feedback phase compared to the information of production and distribution of phase is more social, so its application is more rational, including data mining and knowledge discovery, analysis of emotional and social computing, question answering system, the human-computer interaction system, etc. The existing analysis of information values in the age of intelligence often thinks that the bias of values is embedded in the algorithm.Algorithms become something of a magical mystery. In fact, algorithms are a set of "coding processes that convert input data into desired output based on some specified calculation."Like recipes, algorithms provide a way to convert data into simple or complex products.Different from human decision making, algorithms are formalized, while the computing theories in embedded algorithms are not "divine" and unexplainable. Theoretically, they can be fully described and repeatedly verified.People rely more and more on artificial intelligence applications. Whether the privacy and emotional needs of the audience can be treated objectively, fairly, fairly and effectively needs to be tackled and overcome in the process of the development of algorithm technology.

\subsection{No security}

This artificial intelligence technology has led to a trend of robots gradually replacing humans, challenging the meaning of human existence. From the perspective of the design of traditional legal system, it is difficult to regulate the potential risks of artificial intelligence technology in advance. At the moment, the main risk of AI technology is manipulation, and for this risk it seems possible to hold the operators themselves accountable. However, in the long run, it is difficult to make any effective legal regulation on the risk of artificial intelligence getting rid of human manipulation. As mentioned above, technology has its own advantages and disadvantages. The key point is how to use technology. If it can be used correctly, it can greatly improve the level of productivity, but it may also be misused, which may cause damage to social legal interests. German scholar Beck believes that modernization is becoming its own theme and problem, so it becomes reflective.

\subsection{Ethics laws}

Most of us use algorithms every day, we rule algorithms every day, and we are ruled by algorithms every day. But most of us don't know much about the algorithm code through which the algorithm set works. This unknown knowledge makes us doubt the mysterious things behind the screen, as well as something fundamental that is different from the goals of our human peers. " In the field of computer science, algorithms are generally regarded as a set of command guidelines for solving specific problems, which will neither comfort nor threaten people. As long as you enter the command correctly and follow the guide, you will get the corresponding consequences. However, on the surface, it seems that technology is playing a role, but in fact, the human factors behind technology are completely covered up.

The ethical and legal issues related to intelligent information recommendation algorithms are also the focus of discussion in the development of artificial intelligence in China. Because the algorithm technology not only has the opacity 
represented by its own trade secrets, but also has the characteristics of black box because of the professionalism of the technology. It is precisely because of the opaque black box nature that it is difficult to define the rights and obligations of algorithm technology. At present, the legal constraint on the ethics of algorithm technology still belongs to the aftereffect type, paying attention to the consequences of algorithm technology while ignoring the constraints on its process, and still stays at the stage of ex post review and disposal, generally waiting until problems are found before taking measures, such as current restriction, post deletion, removal of shelves and so on. In addition, the algorithm technology is complex, and it is difficult for the audience to understand the basis and rules of the recommended information behind the algorithm technology. Therefore, it is difficult to solve the ethical and legal problems brought about by algorithm technology, such as data abuse, discrimination, invasion of privacy, infringement of intellectual property rights and so on. At present, the laws and regulations on algorithm technology are not perfect, such as the protection of all kinds of data caused by the application of algorithm technology. Data belongs to a new type of property rights, the ownership and use of data, the security of data, especially related to national security, there are still some defects, which need to be defined in time at the level of ethics and law. For example, the responsibility provisions of the media platform are not clear enough and other issues need to be improved.

\section{CONCLUSIONS}

From the perspective of sociology of technology, as the core technological route that affects human society and behavior, the study of intelligent technology and social culture and values can also promote the academic research on the relationship between technology and society. Especially in the European academic research in recent years, the network society in the context of information technology has considered media as a social "metaprocess", as if the media logic under the influence of technology has penetrated into politics, economy and other fields. The initiative of the latter is gradually disappearing. The key of artificial intelligence technology + media lies in the core of intelligent technology-returning to value rationality.Due to the application of algorithms to promote new changes in the form of power, the algorithmic society has been formed, and a new round of competition has begun between the state and non-state forces in the era of artificial intelligence.As a technology in the field of computer, algorithm has profoundly changed our lives.However, the social, political and legal problems it raises also go far beyond the technical field itself.

Only by guiding to adhere to the socialist core values, establishing a set of man-machine cooperative information management and distribution mechanism, and gathering value rationality in the process of intelligent production and distribution of information, can people return to themselves.

\section{REFERENCES}

[1] Guo Quanzhong, Guo Fengjuan. Intelligent Communication: the latest Communication Mode of the Evolution of Internet Media in China [J]. Media Review, 2017 (01): 77-79.

[2] Quanyan. Morphological construction and risk control of algorithm communication in smart media era [J]. Nanjing Social Sciences, 2020 (11): 99-107.

[3] Xu Cong Yao. Research on the Design of O 20 products from the Perspective of Intelligent Media [J]. Design, 2020 (21): 50-52.

[4] Liu Haimeng, Gong Fangmin, Gong Zhuiliu. The "human value" turns to $[\mathrm{J}]$ in the news production in the intelligent media era. Today's Media, 2020 (11): 37-million.

[5] Liu Yanping. Reflections on the mainstream value led by the new mainstream media in crisis communication [J]. Today's Media, 2020 (11): 6-9. 